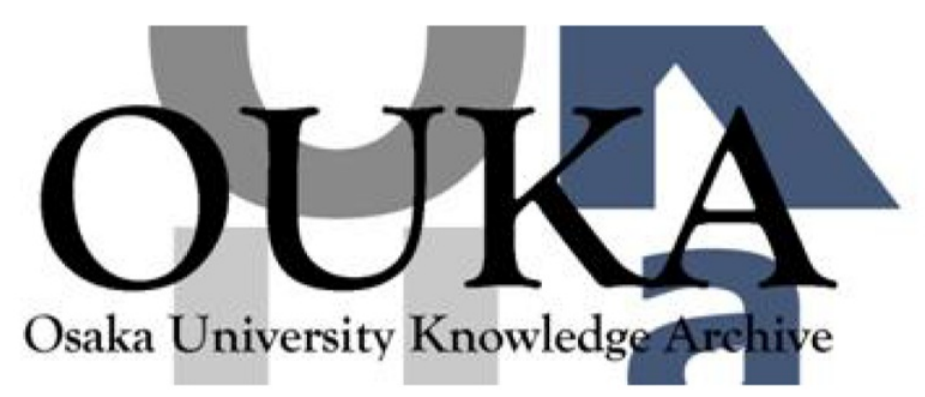

\begin{tabular}{|c|l|}
\hline Title & $\begin{array}{l}\text { Unified explanation of quark and lepton masses } \\
\text { and mixings in the supersymmetric S0(10) model }\end{array}$ \\
\hline Author(s) & $\begin{array}{l}\text { Oda, Kinya; Takasugi, Ei ichi; Tanaka, Minoru et } \\
\text { al. }\end{array}$ \\
\hline Citation & Physical Review D. 59(5) p. 055001 \\
\hline Issue Date & $1999-01-14$ \\
\hline oaire:version & VoR \\
\hline URL & https://hdl. handle.net/11094/78782 \\
\hline rights & ○ 1999 American Physical Society \\
\hline Note & \\
\hline
\end{tabular}

Osaka University Knowledge Archive : OUKA

https://ir. Library. osaka-u. ac. jp/

0saka University 


\title{
Unified explanation of quark and lepton masses and mixings in the supersymmetric $\mathrm{SO}(10)$ model
}

\author{
Kin-ya Oda, ${ }^{*}$ Eiichi Takasugi, ${ }^{\dagger}$ Minoru Tanaka, ${ }^{\dagger}$ and Masaki Yoshimura ${ }^{\S}$ \\ Department of Physics, Osaka University, Toyonaka, Osaka 560, Japan
}

(Received 6 August 1998; published 14 January 1999)

\begin{abstract}
We discussed neutrino masses and mixings in the SUSY SO(10) model where quarks and leptons have Yukawa couplings to at least two 10 and one $\overline{126}$ Higgs scalars. In this model, the Dirac and the right-handed Majorana mass terms are expressed by linear combinations of quark and charged lepton mass matrices, which then determine the neutrino mass matrix by the seesaw mechanism. We show that there are various solutions to reproduce a large mixing angle for $\nu_{\mu} \nu_{\tau}$ and a small mixing angle for $\nu_{e^{-}} \nu_{\mu}$, as well as the hierarchical mass spectrum of neutrinos. [S0556-2821(99)07101-5]
\end{abstract}

PACS number(s): 12.10.Dm, 12.15.Ff, 12.60.Jv, 14.60.Pq

\section{INTRODUCTION}

The Super-Kamiokande group announced a remarkable report [1] of evidence neutrino oscillations and neutrino masses based on atmospheric neutrino observations. The $\nu_{\mu}-\nu_{e}$ oscillation scenario is excluded by the $\mathrm{CHOOZ}$ data [2] and also the Super-Kamiokande data [1], and $\nu_{\mu}-\nu_{\tau}$ oscillation is favored, although the interpretation of the $\nu_{\mu}-\nu_{\text {sterile }}$ is possible. Within the three-neutrino scenario, they showed that $\sin ^{2} 2 \theta_{\nu \tau}>0.8$ and $\Delta m_{23}^{2}$ is in the range $10^{-3}-10^{-2} \mathrm{eV}^{2}$. On the other hand, from observation of the day and night difference of the solar neutrino flux, it seems that small mixing for $\nu_{e}-\nu_{\mu}$ is favored for the solar neutrino problem [3].

In this paper, we consider these facts seriously and seek a scenario to reproduce the pattern of neutrino mixings and the neutrino mass spectrum. In particular, we aim at how the large mixing between $\nu_{\mu}$ and $\nu_{\tau}$ arises, while one keeps the small mixing between $\nu_{e}$ and $\nu_{\mu}$ in the framework of supersymmetric (SUSY) SO(10) grand unified theory (GUT) models. We consider the model where fermions have Yukawa couplings to at least two 10 and one $\overline{126}$ Higgs scalars. In this scenario, the Dirac and right-handed Majorana mass terms are expressed by linear combinations of quark and charged lepton mass matrices and thus the neutrino mass matrix arising from the seesaw mechanism is also determined by quark and charged lepton mass matrices. In the basis where the $u$-type quark mass matrix is diagonal, the $d$-type and also the $l$-type (charged lepton) mass matrices are expected to be almost diagonal so that it is a nontrivial problem to obtain the nonhierarchical neutrino mass matrix for the part related to the second and third generations by using these hierarchical mass matrices, which is needed to obtain the large mixing between $\nu_{\mu}$ and $\nu_{\tau}$.

The model which we consider has been discussed intensively to get the unified description of quark and lepton

\footnotetext{
*Email address: oda@phys.wani.osaka-u.ac.jp

${ }^{\dagger}$ Email address: takasugi@phys.wani.osaka-u.ac.jp

†Email address: tanaka@phys.wani.osaka-u.ac.jp

${ }^{\S}$ Email address: masaki@ phys.wani.osaka-u.ac.jp
}

masses and mixings. Babu and Mohapatra [4] and Lee and Mohapatra [5] considered the minimal (SUSY) SO(10) GUT model, where quarks and leptons have Yukawa couplings to only one 10 and one $\overline{126}$ Higgs scalars, in order to get predictions of neutrino masses and mixings. Along this line, the texture-zero analysis based on models of one 10 and two or three $\overline{126}$ Higgs scalars was made by Babu and Shafi [6] and Achiman and Greiner [7]. All these models have predicted a small mixing angle for $\nu_{\mu}-\nu_{\tau}$. The particular interest of these models lies in the fact that neutrino mixings and ratios of neutrino masses are predicted.

Recently, Brahmachari and Mohapatra [8] discussed that minimal SUSY SO(10) models with one 10 and one $\overline{126}$ are unable to predict a large mixing angle for $\nu_{\mu}-\nu_{\tau}$. Therefore, they considered SUSY SO(10) models where Higgs multiplets are in two 10 and one $\overline{126}$ representations. By considering a type-II seesaw mechanism where the neutrino mass matrix consists of the left-handed Majorana mass term and the seesaw term, they found a solution which predicts a large mixing angle for $\nu_{\mu}-\nu_{\tau}$ and a small mixing angle for $\nu_{e}-\nu_{\mu}$.

Motivated by the work in Ref. [8], we consider SUSY $\mathrm{SO}(10)$ models where quarks and leptons have Yukawa couplings to more than two 10 and one $\overline{126}$ Higgs scalars. The model is essentially the same as that by Brahmachari and Mohapatra except that we do not consider the left-handed Majorana mass term. We assume that the low energy theory of these models is the minimal supersymmetric standard model (MSSM) with two Higgs doublets which are linear combinations of the doublets in the 10 's and the $\overline{126}$. We assume that $H_{u}=\alpha_{1} H_{u}\left(10_{1}\right)+\alpha_{2} H_{u}\left(10_{2}\right)+\alpha_{3} H_{u}(\overline{126})$ and $H_{d}=\beta_{1} H_{d}\left(10_{1}\right)+\beta_{2} H_{d}\left(10_{2}\right)+\beta_{3} H_{d}(\overline{126})$. Quark and lepton masses come from the Yukawa couplings,

$$
W_{Y}=h_{i, a b} \psi_{a} \psi_{b} H_{i}(10)+f_{a b} \psi_{a} \psi_{b} \bar{\Delta}(1 \overline{2} 6),
$$

where $\psi_{a}$ is the 16-dimensional fermions with the family index $a$. The matrices $h_{j}$ 's and $f$ are $3 \times 3$ complex symmetric matrices. Quark and lepton mass matrices are given by

$$
M_{u}=\sum_{j}^{n} v_{u j} h_{j}+\kappa_{u} f, \quad M_{d}=\sum_{j}^{n} v_{d j} h_{j}+\kappa_{d} f,
$$




$$
\begin{gathered}
M_{\nu D}=\sum_{j}^{n} v_{u j} h_{j}-3 \kappa_{u} f, \quad M_{l}=\sum_{j}^{n} v_{d j} h_{j}-3 \kappa_{d} f, \\
M_{\nu R}=v_{R} f,
\end{gathered}
$$

where $v_{u 1}$ is the vacuum expectation value of $H_{u}\left(10_{1}\right)$ multiplied by the ratio of $H_{u}$ in $H_{u}\left(10_{1}\right)$. Others are defined similarly. They satisfy

$$
\sqrt{\sum_{j}^{n}\left(v_{u j}^{2}+v_{d j}^{2}\right)+\kappa_{u}^{2}+\kappa_{d}^{2}}=246 \mathrm{GeV} .
$$

The parameter $v_{R}$ is the scale of the right-handed Majorana neutrino masses.

From Eq. (2), we obtain the relation for $n \geqslant 2$ :

$$
\begin{aligned}
& M_{\nu D}=M_{u}-r\left(M_{d}-M_{l}\right), \\
& M_{\nu R}=R\left(M_{d}-M_{l}\right),
\end{aligned}
$$

where $r=\kappa_{u} / \kappa_{d}$ and $R=v_{R} / 4 \kappa_{d}$. Since we consider that the neutrino mass matrix $m_{\nu}$ is derived by the seesaw mechanism as $m_{\nu}=-M_{\nu D}^{T} M_{\nu R}^{-1} M_{\nu D}, m_{\nu}$ is essentially determined by quark and charged lepton mass matrices. The only other parameters are the ratios of vacuum expectation values, $r$ and $R$. The parameter $R$ is used to control the overall normalization of neutrino masses, and therefore $r$ is the only adjusting parameter to fix neutrino mixings and the ratios of neutrino masses. The models presented above are quite tight so that there needs to be some mechanism which naturally leads to large mixing between $\nu_{\mu}$ and $\nu_{\tau}$.

The topics in this paper are as follows

(i) We show that the minimal model consisting of one 10 and one $\overline{126}$ Higgs is excluded. This is because of the inability to reproduce the experimentally observed pattern of the mass spectrum of $d$-type quarks and charged leptons simultaneously. Our reason is severer than the reason raised by Brahmachari and Mohapatra [8]. They argued that the model is unable to predict a large mixing angle between $\nu_{\mu}-\nu_{\tau}$ and thus the model is rejected. The outline of our discussion is given in the Appendix.

(ii) We consider the (type-I) seesaw mechanism to obtain the neutrino mass matrix in contrast to Brahmachari and Mohapatra [8] who used the type-II seesaw mechanism where the left-handed Majorana mass term is added to the seesaw term. We simply avoid introducing an extra freedom due to the left-handed Majorana mass term.

(iii) We give a qualitative argument on how to get the less hierarchical structure of the neutrino mass matrix which is derived through the seesaw mechanism by using the Dirac and right-handed Majorana mass terms which are given as linear combinations of hierarchical $u$-type, $d$-type, and the charged lepton mass terms.

(iv) We found many possible ranges of the parameter $r$ to lead a small mixing for $\nu_{e}-\nu_{\mu}$ and a large mixing for $\nu_{\mu}-\nu_{\tau}$ as well as the hierarchical neutrino mass spectrum. Each region corresponds to slightly different mixing angles and neutrino masses.
The paper is organized as follows: In Sec. II, we explain the general structure of the Dirac mass matrix and the Majorana mass matrix, and then we explaine the strategy how to obtain the large mixing between $\nu_{\mu}$ and $\nu_{\tau}$. In Sec. III, we present a numerical analysis on whether models can reproduce the atmospheric neutrino as well as the solar neutrino experimental data. A summary is given in Sec. IV.

\section{UNIFIED DESCRIPTION OF QUARK AND LEPTON MASSES AND MIXINGS}

In this section, we present a qualitative argument on how to derive the large mixing between $\nu_{\mu}$ and $\nu_{\tau}$.

\section{A. Basis of mass matrices}

There is a freedom in choosing the basis of mass matrices. Because neutrino mass matrices are expressed as linear combinations of quark and charged lepton mass matrices, we can transform all mass matrices simultaneously by a unitary matrix such that $M_{k}^{\prime} \equiv U^{T} M_{k} U$, where $k=u, d, l, \nu D, \nu R$ by a unitary matrix $U$. Since all mass matrices are symmetric ones, we choose $U$ such that it diagonalizes $M_{u}$ :

$$
M_{u}^{\prime} \equiv U^{T} M_{u} U=D_{u},
$$

where $D_{u}=\operatorname{diag}\left(\left|m_{u}\right|,\left|m_{c}\right|,\left|m_{t}\right|\right)$. Next, we introduce a unitary matrix $D$ which diagonalizes $M_{d}$, i.e., $D^{T} M_{d} D=D_{d}$, with $D_{d}=\operatorname{diag}\left(\left|m_{d}\right|,\left|m_{s}\right|,\left|m_{b}\right|\right)$. By using $U$ and $D$, the general form of the Cabibbo-Kobayashi-Maskawa (CKM) matrix is given by $U^{\dagger} D=\phi_{u}^{\dagger} K \phi_{d}$, where $K$ is a special form of the CKM matrix and $\phi_{i}(i=u, d)$ are diagonal phase matrices. By using $K$ and phase matrices, $M_{d}^{\prime}$ is expressed by

$$
M_{d}^{\prime}=U^{T} M_{d} U=U^{T} D^{*} D_{d} D^{\dagger} U=\phi_{u}^{T} K^{*} \phi_{d}^{*} D_{d} \phi_{d}^{*} K^{\dagger} \phi_{u} .
$$

Finally, we transform all matrices by the phase matrix $\phi_{u}$ as $\phi_{u}^{*} M_{k}^{\prime} \phi_{u}^{\dagger} \equiv \widetilde{M}_{k}$. This is the basis which we use in

$$
\widetilde{M}_{u}=\widetilde{D}_{u}, \quad \widetilde{M}_{d}=K^{*} \widetilde{D}_{d} K^{\dagger},
$$

and $\quad \tilde{M}_{l}, \quad \tilde{M}_{\nu D}, \quad$ and $\quad \tilde{M}_{\nu R}$, where $\widetilde{D}_{u}=\phi_{u}^{*} D_{u} \phi_{u}^{\dagger}$ $\equiv \operatorname{diag}\left(m_{u}, m_{c}, m_{t}\right)$ and $\widetilde{D}_{d}=\phi_{d}^{*} D_{d} \phi_{d}^{\dagger} \equiv \operatorname{diag}\left(m_{d}, m_{s}, m_{b}\right)$. In this basis, all quark masses are complex quantities. Now we have

$$
\widetilde{M}_{\nu D}=\widetilde{M}_{u}-r \tilde{M}, \quad \tilde{M}_{\nu R}=R \tilde{M},
$$

where

$$
\widetilde{M}=\widetilde{M}_{d}-\tilde{M}_{l} .
$$

The left-handed neutrino mass matrix $m_{\nu}$ is given by the seesaw mechanism as

$$
m_{\nu}=-\tilde{M}_{\nu D}^{T}\left(\tilde{M}_{\nu R}\right)^{-1} \widetilde{M}_{\nu D} .
$$

Thus the neutrino mass matrix is essentially determined once quark and charged lepton mass matrices are given. The only 
other parameters in the model are $r$ and $R$. The parameter $R$ determines the overall scale of the neutrino masses. Thus the parameter $r$ is the only adjusting parameter to reproduce the desired neutrino mixing angles and neutrino mass ratios.

\section{B. Quark and charged lepton masses and CKM mixings at the GUT scale}

We use the following quark and charged lepton masses, and the CKM mixing angles at the GUT scale $\left(2 \times 10^{16} \mathrm{GeV}\right)$, which were estimated in the minimal supersymmetric standard model with $\tan \beta=10$ following Fusaoka and Koide [9]:

$$
\begin{aligned}
& \left|m_{u}\right|=0.00104, \quad\left|m_{c}\right|=0.302, \quad\left|m_{t}\right|=129, \\
& \left|m_{d}\right|=0.00133, \quad\left|m_{s}\right|=0.0265, \quad\left|m_{b}\right|=1.00,
\end{aligned}
$$

$$
\left|m_{e}\right|=0.000325, \quad\left|m_{\mu}\right|=0.0686, \quad\left|m_{\tau}\right|=1.171,
$$

$\sin \theta_{12}=-0.2205, \quad \sin \theta_{13}=0.0026, \quad \sin \theta_{23}=0.0318$,

where fermion masses are defined in units of GeV. Although these values of the parameters should have errors, we neglect errors since our purpose is to answer whether the parameter ranges of $r$ exist to reproduced the desired neutrino mixings and neutrino spectrum. The above values of CKM mixings at the GUT scale are given by taking into account the one-loop contribution by keeping only $m_{t}$ and $m_{b}$.

In the numerical analysis, we use the values in Eqs. (11) and (12). For the qualitative analysis, we use the Wolfenstein form for the CKM matrix $K$ at the GUT scale:

$$
K=\left(\begin{array}{ccc}
1-\frac{1}{2} \lambda^{2}-\frac{1}{8} \lambda^{4} & \lambda & \Lambda \lambda^{4} e^{-i \delta} \\
-\lambda+\frac{1}{2} A^{2} \lambda^{5} & 1-\frac{1}{2} \lambda^{2}-\left(\frac{1}{8}+\frac{1}{2} A^{2}\right) \lambda^{4} & A \lambda^{2} \\
A \lambda^{3}-\Lambda \lambda^{4} e^{i \delta} & -A \lambda^{2}+\frac{1}{2} A \lambda^{4}-\Lambda \lambda^{5} e^{i \delta} & 1-\frac{1}{2} A^{2} \lambda^{4}
\end{array}\right)+O\left(\lambda^{6}\right)
$$

where $\lambda=0.2205, A=0.6540$, and $\Lambda=1.100$ by using the mixing angles in Eq. (12).

\section{Explicit form of quark mass matrices at the GUT scale}

Because of the hierarchy of magnitudes of quark masses, we parametrize $\widetilde{D}_{u}$ and $\widetilde{D}_{d}$ as follows:

$$
\widetilde{D}_{u}=m_{t}\left(\begin{array}{ccc}
\xi_{u t} \lambda^{7} & & \\
& \xi_{c t} \lambda^{4} & \\
& & 1
\end{array}\right), \quad \widetilde{D}_{d}=m_{b}\left(\begin{array}{ccc}
\xi_{d b} \lambda^{4} & \\
& \xi_{s b} \lambda^{2} & \\
& & 1
\end{array}\right),
$$

where $\xi$ 's are quantities of order unity. From Eq. (11), we have $\left|\xi_{u t}\right|=0.318,\left|\xi_{c t}\right|=0.990,\left|\xi_{d b}\right|=0.563$, and $\left|\xi_{s b}\right|=0.545$.

By using them, one finds

$$
\widetilde{M}_{d}=m_{b}\left(\begin{array}{ccc}
\left(\xi_{d b}+\xi_{s b}\right) \lambda^{4} & \xi_{s b} \lambda^{3}-\left(\xi_{d b}+\frac{1}{2} \xi_{s b}\right) \lambda^{5} & \Lambda \lambda^{4} e^{-i \delta}-\xi_{s b} A \lambda^{5} \\
\xi_{s b} \lambda^{3}-\left(\xi_{d b}+\frac{1}{2} \xi_{s b}\right) \lambda^{5} & \xi_{s b} \lambda^{2}+\left(-\xi_{s b}+A^{2}\right) \lambda^{4} & A \lambda^{2}-\xi_{s b} A \lambda^{4} \\
\Lambda \lambda^{4} e^{-i \delta}-\xi_{s b} A \lambda^{5} & A \lambda^{2}-\xi_{s b} A \lambda^{4} & 1-A^{2} \lambda^{4}
\end{array}\right)+\left(\begin{array}{ccc}
O\left(\lambda^{6}\right) & O\left(\lambda^{6}\right) & O\left(\lambda^{7}\right) \\
O\left(\lambda^{6}\right) & O\left(\lambda^{6}\right) & O\left(\lambda^{6}\right) \\
O\left(\lambda^{7}\right) & O\left(\lambda^{6}\right) & O\left(\lambda^{6}\right)
\end{array}\right) .
$$

It is interesting to observe the difference between the mass hierarchy of $u$-type quarks and that of $d$-type quarks. While $\left|m_{u} / m_{t}\right| \sim O\left(\lambda^{7}\right)$ and $\left|m_{c} / m_{t}\right| \sim O\left(\lambda^{4}\right), \quad\left|m_{d} / m_{b}\right|$ $\sim O\left(\lambda^{4}\right)$ and $\left|m_{s} / m_{b}\right| \sim O\left(\lambda^{2}\right)$. That is, the mass hierarchy of $d$ quarks is much less severe than that of $u$ quarks. Next, we observe that $\left(\tilde{M}_{d}\right)_{22} \sim\left(\tilde{M}_{d}\right)_{23} \sim O\left(\lambda^{2}\right)$. These are crucial in the following discussions.

\section{Hierarchy in the neutrino mass matrix}

First, we discuss what kind of neutrino mass matrix is required from the recent data. From the data $[1,2,3]$, the neutrino mass mixing matrix $O$ is almost fixed aside from $C P$ violation phases as

$$
O \sim\left(\begin{array}{ccc}
1 & \epsilon & \epsilon^{\prime} \\
-\epsilon & c & s \\
\epsilon^{\prime} & -s & c
\end{array}\right)
$$

where $s=\sin \theta_{\mu \tau}$ and $c=\cos \theta_{\mu \tau}$, with $\theta_{\mu \tau}$ is the mixing angle between $\nu_{\mu}$ and $\nu_{\tau}$ neutrinos, $\epsilon \sim \lambda^{2}$ and $\epsilon^{\prime} / \epsilon \sim s /(1$ $+c)$. From this mixing matrix, the expected neutrino mass matrix is given by

$$
m_{\nu}=O D_{\nu} O^{T} \sim m_{\nu_{\tau}}\left(\begin{array}{ccc}
r_{1}+\epsilon^{\prime 2} & s \epsilon^{\prime} & c \epsilon^{\prime} \\
s \epsilon^{\prime} & s^{2}+r_{2} c^{2} & s c \\
c \epsilon^{\prime} & s c & r_{2} s^{2}+c^{2}
\end{array}\right),
$$


where $r_{1}=m_{\nu e} / m_{\nu \mu}, \quad r_{2}=m_{\nu \mu} / m_{\nu \tau} \sim \pm 1 / 10$. If we take $\sin ^{2} 2 \theta_{\mu \tau} \geqslant 0.7$ for the experimentally allowed region, we see that the submatrix relevant to $\nu_{\mu}$ and $\nu_{\tau}$ should have less hierarchical structure than quark mass matrices as

$$
\begin{aligned}
& \left(\begin{array}{cc}
0.23+0.77 r_{2} & 0.42 \\
0.42 & 0.23 r_{2}+0.77
\end{array}\right) \sim \frac{1}{2}\left(\begin{array}{cc}
1+r_{2} & 1 \\
1 & r_{2}+1
\end{array}\right) \\
& \sim\left(\begin{array}{cc}
0.77+0.23 r_{2} & 0.42 \\
0.42 & 0.77 r_{2}+0.23
\end{array}\right) .
\end{aligned}
$$

The above matrices show variations of their components. They corresponds to the angles $\theta_{\mu \tau}=\theta_{0}, \pi / 4$, and $\pi / 2-\theta_{0}$ with $\sin ^{2} 2 \theta_{0}=0.7$. From the above analysis, we observe the following: (1) The 1-2 and 1-3 elements are of order $\lambda^{2}$, while the $1-1$ element is of order $\lambda^{4}$. (2) In general, the nonhierarchical structure appears for the part related to $\nu_{\mu}$ and $\nu_{\tau}$. Even in the extreme case, the hierarchy is at most of order $\lambda$.

\section{E. Mechanism which leads the large mixing between $\nu_{\mu}$ and $\nu_{\tau}$}

In the basis where $\tilde{M}_{u}$ is diagonal, $\tilde{M}_{d}$ takes a hierarchical form. Then it is natural to suppose that $\tilde{M}_{l}$ also takes a hierarchical form. On the other hand, in order to get the nonhierarchical neutrino mass matrix for the part related to $\nu_{\mu}$ and $\nu_{\tau}$, at least one of $M_{\nu D}$ and $M_{\nu R}$ should take the nonhierarchical form for the relevant part. Since $M_{\nu D}$ and $M_{\nu R}$ are linear combinations of hierarchical mass matrices $\widetilde{M}_{u}, \widetilde{M}_{d}$, and $\widetilde{M}_{l}$, there needs to be some mechanism to get the nonhierarchical structure for $M_{\nu D}$ and/or $M_{\nu R}$ for the relevant part. This is a necessary condition and does not imply the desired form of neutrio mass is obtained. However, we seek this possibility.

The hint lies in the fact that the 2-2 element of $\tilde{M}_{d}$ is the same size as the 2-3 element. We consider how to obtain the nonhierarchical form of $\widetilde{M}_{\nu D}$. By adjusting $r$, we make the 3-3 element of $\tilde{M}_{\nu D}=\widetilde{M}_{u}-r \tilde{M}$ as small as of order $\lambda^{2} m_{t}$. Then $m_{c}$ and $m_{u}$ do not contribute to $\tilde{M}_{\nu D}$ because of the large hierarchy of $u$-type quark masses. Thus only $m_{t}$ in $\tilde{M}_{u}$ contributes to $\tilde{M}_{\nu D}$. Thus the nonhierarchical structure arises with the above condition for the 3-3 element.

We consider the above condition in detail, which is treated as two separate cases.

\section{1. $m_{b} m_{\tau}<0$ case}

In this case, $\tilde{M}_{\nu R}$ has a hierarchical form as we see from Eqs. (8) and (9). We require that $\tilde{M}_{\nu D}$ have a nonhierarchical form for the relevant part. This is achieved by requiring that the 3-3 element of $\tilde{M}_{\nu D}$ to be of order of the 2-3 element:

$$
m_{t}-r\left(m_{b}-m_{\tau}\right) \sim O\left(\lambda^{2} m_{t}\right) .
$$

That is, the value of $r$ is fixed to be of order

$$
r \sim \frac{m_{t}}{m_{b}-m_{\tau}} \sim 50
$$

In particular, the range of $r$ which gives a nonhierarchical neutrino mass structure will consist of two parts. One is the region $r<50$, and the other is the one $r>50$, because the exact equality $m_{t}=r\left(m_{b}-m_{\tau}\right)$ gives the vanishing 3-3 element of the neutrino mass matrix so that we cannot reproduce the desired mass matrix given in Eq. (18).

$$
\text { 2. } m_{b} m_{\tau}>0 \text { case }
$$

In this case, $\widetilde{M}_{\nu R}$ has a nonhierarchical form due to the cancellation between $m_{b}$ and $m_{\tau}$. There are two cases.

$a$. The case where $\tilde{M}_{\nu D}$ has a nonhierarchical form

$$
r \sim \frac{m_{t}}{m_{b}-m_{\tau}} \sim 750 .
$$

The range of $r$ which gives a nonhierarchical neutrino mass structure will consist of two parts, the one $r<750$ and the other $r>750$.

$b$. The case where $\tilde{M}_{\nu D}$ has a hierarchical form

$$
r \ll \frac{m_{t}}{m_{b}-m_{\tau}} .
$$

The above conditions are of course only the necessary ones to achieve a nonhierarchical form of the neutrino mass matrix. The problem is whether there are regions of $r$ which reproduce the desired neutrino mixing angles and more importantly the hierarchical spectrum of neutrino masses. There is no guarantee of the existence of such a parameter region of $r$. We have to calculate the neutrino matrix for a given $r$ and examine whether the desired mixings and mass ratios are realized, especially by paying special attention to the abovementioned parameter regions of $r$.

\section{ANALYSIS}

Before the analysis, the following comments are in order.

(i) The parameters. In addition to $r$ and $R$, we have sign freedoms of quark and lepton masses. Since one of the phase can be fixed, we choose $m_{b}>0$. If we scale $\tilde{M}_{\nu D}$ by $m_{t}$, the parameter $r$ enters as $r / m_{t}$. Thus we can fix $r>0$, while we allow $m_{t}$ to take both positive and negative signs. Thus we fix $m_{b}>0$ and $r>0$ and take all combinations of signs of other fermion masses.

(ii) The desired neutrino mixings and masses. We consider the following constraints on ranges of neutrino mixings and neutrino masses $[1,10]$ :

$$
\begin{gathered}
3 \times 10^{-3}<\sin ^{2} 2 \theta_{e \mu}<2.0 \times 10^{-2}, \quad 0.7<\sin ^{2} 2 \theta_{\mu \tau}, \\
3 \times 10^{-4}<\frac{\Delta m_{12}^{2}}{\Delta m_{23}^{2}}<5 \times 10^{-2},
\end{gathered}
$$

where we used $3 \times 10^{-6} \mathrm{eV}^{2}<\Delta m_{12}^{2}<1 \times 10^{-5} \mathrm{eV}^{2}$ and $2 \times 10^{-4} \mathrm{eV}^{2}<\Delta m_{23}^{2}<1 \times 10^{-2} \mathrm{eV}^{2}$. Since we are dealing with the hierarchical mass spectrum of neutrino case, the overall normalization is fixed by the mass $m_{\nu \tau}$. The parameter $R$ is determined to fix $m_{\nu \tau}$ in the range of 


$$
\begin{aligned}
& 3 \times 10^{-6} \mathrm{eV}^{2}<\Delta m_{12}^{2}<1 \times 10^{-5} \mathrm{eV}^{2}, \\
& 2 \times 10^{-4} \mathrm{eV}^{2}<\Delta m_{23}^{2}<5 \times 10^{-3} \mathrm{eV}^{2} .
\end{aligned}
$$

In the above, we defined $\Delta m_{j k}^{2} \equiv m_{k}^{2}-m_{j}^{2}$ and $m_{1}^{2} \ll m_{2}^{2}$ $\ll m_{3}^{2}$.

(iii) The mixing angles. Since we are looking for the solutions which reproduce the hierarchical neutrino mass spectrum and the mixings in Eqs. (23) and (24), we may treat the three-neutrino mixing as if it is due to the two-neutrino mixing. In other words, we may define angles by $\sin \theta_{e \mu}$ $\simeq\left(U_{\nu}\right)_{12}$ and $\sin \theta_{\mu \tau} \simeq\left(U_{\nu}\right)_{23}$, where $U_{\nu}$ is the neutrino mixing matrix. With this approximation, we seek the range of parameter $r$ which reproduce the desired mixings and ratios of masses to solve the atmospheric neutrino problem and the solar neutrino problem. Once we find the solution, then we examine whether the mixings and masses that we obtained do not violate the CHOOZ bound for the $\bar{\nu}_{e}-\bar{\nu}_{X}$ oscillation [2]. Since $\Delta m_{12}^{2} \ll \Delta m_{23}^{2} \simeq \Delta m_{13}^{2} \simeq m_{3}^{2}$, we can treat the mixing angle as $\sin \theta_{e X}=\left(U_{\nu}\right)_{13}$ and the mass-squared difference as $\Delta m^{2} \simeq m_{3}^{2}$ in our three-neutrino mixing scenario.

\section{A. Simplification of the problem}

In this paper, we use the following simplification.

(i) The $C P$ violation is neglected. Explicitly, we perform the numerical analysis by setting the Kobayashi-Maskawa $\mathrm{CP}$ violation angle $\delta$ to zero and taking the quark and charged lepton masses as real. However, we set the signs of fermion masses free so that we have to consider all combinations of signs of quark and charged lepton masses.

(ii) $\widetilde{M}_{l}$ is assumed to be diagonal in the basis we adopted in this paper.

With these simplifications, the neutrino mass matrix $m_{\nu}$ is determined by quark and charged lepton masses, CKM mixing angles, $r$ and $R$. The parameter $R$ determines the overall scale of $\tilde{M}_{\nu R}$ so that it plays a role of adjusting the overall scale of neutrino masses in the seesaw mechanism. The neutrino mixing angles and the ratios of neutrino masses are solely determined by only one parameter $r$. Thus the present model is a quite tightly constrained one.

Once the ranges of the parameter $r$ which reproduce the desired neutrino mixing angles and ratios of masses are found, the introduction of $\mathrm{CP}$ violation will relax the region of $r$ and $R$. The same is expected if we relax (ii). Therefore, in this paper we concentrate on finding the region of $r$ and $R$ in the simplified and tight situation.

\section{B. Result}

The procedure of our analysis is as follows: For a given $r>0$ and a combination of signs of fermion masses, $\tilde{M}_{\nu D}$ and $\widetilde{M}_{\nu R} / R$ are calculated. We compute the neutrino mass matrix by the seesaw mechanism. Then we compute neutrino mixings and neutrino mass ratios to see whether the results reproduce the desired ones.

We are fortunate that we found many regions of $r$ which reproduce the desired mixings and masses. The results are summarized in Tables I and II.

In Table I(a), we show the result for $m_{b} m_{\tau}<0$. Solutions exist for various combinations of signs of quark and charged lepton as we see from the table. Each sign combination is expressed by the abbreviated notation $E i-D j-U k X$. The indices $i, j$, and $k$ run $1,2,3$, and 4 , which express signs of masses as $(-,-),(-,+),(+,-)$, and $(+,+)$ for a pair $(e, \mu)$ (denoted by $E$ ), $(d, s)$ (denoted by $D$ ), and $(u, c)$ (denoted by $U$ ), respectively, and $X$ takes $P$ and $M$, which means that the sign of $m_{t}$ is positive and negative, respectively. The region of $r$ which reproduces the data in Eq. (23) is given in the column $r$. The column $\sin ^{2} 2 \theta_{\mu \tau}$ shows the largest value of it, which is achieved by a specific value of $r$ shown in the column $R\left(10^{13}\right)(r)$. The value of $R$ fixes the overall normalization of the neutrino mass, especially $m_{\nu \tau}$. We found solutions only for $m_{\tau}<0$ in the range of $r=54-64$, which coincide with our expectation given in Eq. (20). For all cases $\widetilde{M}_{\nu D}$ has a nonhierarchical form, while $\tilde{M}_{\nu R} \equiv R \tilde{M}$ takes a hierarchical form. The mixings and masses with specific values of $r$ and $R$ for all cases are given in Table $\mathrm{I}(\mathrm{b})$.

In Table II(a), we show the result for $m_{b} m_{\tau}>0$ case. The notation to discriminate models which differ by sign combinations of fermion masses is the same as in Table I(a). We see that for all solutions $m_{t}<0$ and $r \sim(500-960)$, except one case $(E 1-D 2-U 2 P)$. These cases coincide what we expected from Eq. (21) and both $\widetilde{M}_{\nu D}$ and $\widetilde{M}_{\nu R} \equiv R \tilde{M}$ take non (less) hierarchical forms.

The case $E 1-D 2-U 2 P$ is realized when $r \sim 35$, which is also what we expected in Eq. (22), although the value of $r$ is unknown. In this case, $\widetilde{M}_{\nu D}$ has a hierarchical form, while $\widetilde{M}_{\nu R} \equiv R \tilde{M}$ takes a non (less) hierarchical form. In Table II(b), we show the mixings and masses for all cases.

In the following, the neutrino mass matrix and the neutrino mixing matrix are showed for some typical cases.

\section{1. $m_{b} m_{\tau}<0$ case}

a. E1-D3-U2P with $r=58.2$. The Dirac mass term and the Majorana mass term are given by

$$
\begin{aligned}
& \tilde{M}_{\nu D}=\left(\begin{array}{ccc}
-0.0190 & -0.3530 & -0.1402 \\
-0.3530 & -2.2871 & -1.8954 \\
-0.1402 & -1.8954 & 2.6852
\end{array}\right), \\
& \tilde{M}_{\nu R}=R\left(\begin{array}{lll}
0.0003 & 0.0061 & 0.0024 \\
0.0061 & 0.0445 & 0.0326 \\
0.0024 & 0.0326 & 2.1704
\end{array}\right) .
\end{aligned}
$$

As we expected, $M_{\nu D}$ has a nonhierarchical form for the part relevant to the second and the third generations, while $M_{\nu R}$ is hierarchical, because there is no cancellation of the 3-3 element of $M_{\nu R}$. The neutrino mass matrix and the neutrino mixing matrix are given by

$$
m_{\nu}=-\frac{1}{R}\left(\begin{array}{lll}
1.165 & 20.46 & 8.402 \\
20.46 & 114.3 & 106.7 \\
8.402 & 106.7 & 59.82
\end{array}\right)
$$




$$
U_{\nu}=\left(\begin{array}{ccc}
0.964 & 0.067 & -0.256 \\
-0.242 & 0.613 & -0.752 \\
0.107 & 0.787 & 0.608
\end{array}\right) \text {. }
$$

Then we have $\sin ^{2} 2 \theta_{e \mu}=0.018$ and $\sin ^{2} 2 \theta_{\mu \tau}=0.98$. With $R=1.09 \times 10^{13}$, we found

$$
\begin{aligned}
& m_{1}=-3.22 \times 10^{-5} \mathrm{eV}, m_{2}=2.25 \times 10^{-3} \mathrm{eV}, \\
& m_{3}=-1.83 \times 10^{-2} \mathrm{eV},
\end{aligned}
$$

which are multiplied by $1.09 \times 10^{13} / R$ if the $R$ dependence is kept. Thus

$$
\Delta m_{12}^{2}=5.08 \times 10^{-6} \mathrm{eV}^{2}, \quad \Delta m_{23}^{2}=3.30 \times 10^{-4} \mathrm{eV}^{2} .
$$

As we mentioned before, the mixing angles are computed by assigning $\sin \theta_{e \mu}=\left(U_{\nu}\right)_{12}$ and $\sin \theta_{\mu \tau}=\left(U_{\nu}\right)_{23}$. This approximation is reasonable for the mixings and the hierarchical mass spectrum given in Eqs. (26) and (27).

The sensitivity of mixing angles $\sin ^{2} 2 \theta_{\mu \tau}$ and $\sin ^{2} 2 \theta_{e \mu}$ and also the ratio $\Delta m_{12}^{2} / \Delta m_{23}^{2}$ with respect to the parameter $r$ is shown in Figs. 1(a), 1(b), and 1(c) respectively. The angle $\sin ^{2} 2 \theta_{\mu \tau}$ takes values larger than 0.7 for a wider range of $r$. The same holds for the ratio $\Delta m_{12}^{2} / \Delta m_{23}^{2}$. The allowed region of $r$ is essentially fixed by $\sin ^{2} 2 \theta_{e \mu}$ and the region is between 58 and 62 . This situation holds for all cases E1-D3-UiP $(i=1-4)$. We will show a comparison with $\mathrm{CHOOZ}$ data, the disappearance test of $\bar{\nu}_{e}$, at the end of this section.

b. E4-D2-U3P with $r=58.1$. The Dirac mass term and the Majorana mass term are similar to the case given in Sec. III A 1 a, so that we give only the neutrino mass matrix and the neutrino mixing matrix

\begin{tabular}{|c|c|c|c|c|c|}
\hline \multicolumn{6}{|c|}{$E 1-D 3-U i P(i=2,3,4)$} \\
\hline$e, \mu$ & $d, s$ & $u, c, t$ & $r$ & $R\left(10^{13}\right)(r)$ & $\sin ^{2} 2 \theta_{\mu \tau}$ \\
\hline$(-,-)$ & $(+,-)$ & $(-,-),+$ & $58.9-60.9$ & $0.62-0.73(60.9)$ & 0.89 \\
\hline & & $(-,+),+$ & $58.2-61.7$ & $0.78-1.4(58.2)$ & 0.98 \\
\hline & & $(+,-),+$ & $59.3-60.5$ & $0.61-0.78(60.5)$ & 0.89 \\
\hline & & $(+,+),+$ & $58.5-61.4$ & $0.83-1.4(58.5)$ & 0.98 \\
\hline$E 2-D 2$ & $(i=1,2$, & & & & \\
\hline$e, \mu$ & $d, s$ & $u, c, t$ & $r$ & $R\left(10^{13}\right)(r)$ & $\sin ^{2} 2 \theta_{\mu \tau}$ \\
\hline$(-,+)$ & $(-,+)$ & $(-,-),+$ & $55.0,55.8-56.1,61.9-62.3,63.3-63.9$ & $0.58-1.05$ & 0.98 \\
\hline & & $(-,+),+$ & $55.0-55.6,56.4-56.6,61.4-61.7,62.6-63.8$ & $0.62-0.73(61.4)$ & 0.89 \\
\hline & & $(+,-),+$ & $55.0-55.1,55.8-56.1,61.8-62.2,63.2-63.9$ & $0.58-1.05(56.1)$ & 0.98 \\
\hline & & $(+,+),+$ & $55.0-55.7,56.5-56.7,61.3-61.6,62.5-63.7$ & $0.62-0.69(61.3)$ & 0.90 \\
\hline$E 2-D 4$ & $(i=1,2$, & & & & \\
\hline$e, \mu$ & $d, s$ & $u, c, t$ & $r$ & $R\left(10^{13}\right)(r)$ & $\sin ^{2} 2 \theta_{\mu \tau}$ \\
\hline$(-,+)$ & $(+,+)$ & $(-,-),+$ & $55.0-55.1,62.9-63.1,63.8-64.4$ & $0.73-1.32(62.9)$ & 0.96 \\
\hline & & $(-,+),+$ & $55.0-55.2,55.8,62.3-62.4,63.0-64.1$ & $0.64-0.81(62.3)$ & 0.86 \\
\hline & & $(+,-),+$ & $55.1-55.2,62.9-63.1,63.8-64.4$ & $0.54-0.97(55.2)$ & 0.96 \\
\hline & & $(+,+),+$ & $55.0-55.3,55.8-55.9,62.2-62.3,63.0-64.1$ & $0.64-0.75(62.2)$ & 0.89 \\
\hline$E 4-D 2$ & $(i=1,2$, & & & & \\
\hline$e, \mu$ & $d, s$ & $u, c, t$ & $r$ & $R\left(10^{13}\right)(r)$ & $\sin ^{2} 2 \theta_{\mu \tau}$ \\
\hline$(+,+)$ & $(-,+)$ & $(-,-),+$ & $55.3-55.6,56.8-57.7,60.2-61.2,62.6-63.2$ & $0.65-1.17(57.7)$ & 0.99 \\
\hline & & $(-,+),+$ & $55.4-56.2,57.2-57.6,60.4-60.8,61.9-63.1$ & $0.38-0.68(60.8)$ & 0.85 \\
\hline & & $(+,-),+$ & $55.3-55.8,57.0-58.1,59.8-61.0,62.4-63.2$ & $0.62-1.11(58.1)$ & 0.99 \\
\hline & & $(+,+),+$ & $55.4-56.4,57.4-57.8,60.2-60.7,61.8-63.1$ & $0.36-0.64(60.7)$ & 0.85 \\
\hline$E 4-D 4$ & $(i=1,2$, & & & & \\
\hline$e, \mu$ & $d, s$ & $u, c, t$ & $r$ & $R\left(10^{13}\right)(r)$ & $\sin ^{2} 2 \theta_{\mu \tau}$ \\
\hline$(+,+)$ & $(+,+)$ & $(-,-),+$ & $55.2-55.3,62.7-62.9,63.6-64.3$ & $0.54-0.98(55.3)$ & 0.96 \\
\hline & & $(-,+),+$ & $55.0-55.4,55.9-56.0,62.0-62.2,62.8-64.0$ & $0.64-0.72(62.0)$ & 0.89 \\
\hline & & $(+,-),+$ & $55.2-55.3,62.6-62.9,63.6-64.3$ & $0.67-1.21(62.6)$ & 0.97 \\
\hline & & $(+,+),+$ & $55.0-55.4,56.0-56.1,62.0-62.1,62.8-64.0$ & $0.53-0.58(56.1)$ & 0.89 \\
\hline
\end{tabular}

TABLE I. (a) Ranges of the parameters of $r$ and $R$ to predict the desired values of neutrino mixing angles and masses for $m_{b} m_{\tau}<0$. (b) Predictions of neutrino mixing angles and masses for $m_{b} m_{\tau}<0$. All cases correspond to (a).

(a) 
TABLE I. (Continued).

(b)

\begin{tabular}{|c|c|c|c|c|c|c|c|}
\hline $\begin{array}{l}\text { Model } \\
\left(r, R / 10^{13}\right)\end{array}$ & $\sin ^{2} 2 \theta_{e \mu}$ & $\sin ^{2} 2 \theta_{\mu \tau}$ & $m_{1}\left(10^{-3} \mathrm{eV}\right)$ & $m_{2}\left(10^{-3} \mathrm{eV}\right)$ & $m_{3}\left(10^{-2} \mathrm{eV}\right)$ & $\Delta m_{12}^{2}\left(\mathrm{eV}^{2}\right)$ & $\Delta m_{23}^{2}\left(\mathrm{eV}^{2}\right)$ \\
\hline $\begin{array}{l}E 1-D 3-U 1 P \\
(60.9,0.675)\end{array}$ & 0.019 & 0.89 & -0.0250 & 1.89 & -4.08 & $3.56 \times 10^{-6}$ & $1.66 \times 10^{-3}$ \\
\hline $\begin{array}{l}E 1-D 3-U 2 P \\
(58.2,1.09)\end{array}$ & 0.018 & 0.98 & -0.0322 & 2.25 & -1.83 & $5.08 \times 10^{-6}$ & $3.30 \times 10^{-4}$ \\
\hline $\begin{array}{l}E 1-D 3-U 3 P \\
(60.5,0.695)\end{array}$ & 0.020 & 0.89 & -0.00946 & 1.97 & -3.91 & $3.87 \times 10^{-6}$ & $1.52 \times 10^{-3}$ \\
\hline $\begin{array}{l}E 1-D 3-U 4 P \\
(58.5,1.115)\end{array}$ & 0.019 & 0.98 & -0.0231 & 2.35 & -1.80 & $5.51 \times 10^{-6}$ & $3.19 \times 10^{-4}$ \\
\hline $\begin{array}{l}E 2-D 2-U 1 P \\
(56.1,0.815)\end{array}$ & 0.019 & 0.98 & -0.304 & -2.26 & 2.06 & $5.03 \times 10^{-6}$ & $4.21 \times 10^{-4}$ \\
\hline $\begin{array}{l}E 2-D 2-U 2 P \\
(61.4,0.675)\end{array}$ & 0.014 & 0.89 & -0.436 & -1.93 & 4.09 & $3.54 \times 10^{-6}$ & $1.67 \times 10^{-3}$ \\
\hline $\begin{array}{l}E 2-D 2-U 3 P \\
(56.1,0.815)\end{array}$ & 0.015 & 0.98 & -0.289 & -2.25 & 2.06 & $4.98 \times 10^{-6}$ & $4.21 \times 10^{-4}$ \\
\hline $\begin{array}{l}E 2-D 2-U 4 P \\
(61.3,0.655)\end{array}$ & 0.017 & 0.90 & -0.411 & -1.87 & 4.21 & $3.33 \times 10^{-6}$ & $1.77 \times 10^{-3}$ \\
\hline $\begin{array}{l}E 2-D 4-U 1 P \\
(62.9,1.025)\end{array}$ & 0.014 & 0.96 & -1.29 & -2.58 & 2.06 & $4.98 \times 10^{-6}$ & $4.18 \times 10^{-4}$ \\
\hline $\begin{array}{l}E 2-D 4-U 2 P \\
(62.3,0.725)\end{array}$ & 0.010 & 0.86 & -1.77 & -2.63 & 3.92 & $3.80 \times 10^{-6}$ & $1.53 \times 10^{-3}$ \\
\hline $\begin{array}{l}E 2-D 4-U 3 P \\
(55.2,0.755)\end{array}$ & 0.018 & 0.96 & -1.32 & -2.59 & 2.08 & $4.97 \times 10^{-6}$ & $4.28 \times 10^{-4}$ \\
\hline $\begin{array}{l}E 2-D 4-U 4 P \\
(62.2,0.695)\end{array}$ & 0.019 & 0.89 & -1.80 & -2.60 & 4.09 & $3.53 \times 10^{-6}$ & $1.66 \times 10^{-3}$ \\
\hline $\begin{array}{l}E 4-D 2-U 1 P \\
(57.7,0.91)\end{array}$ & 0.020 & 0.99 & -0.0287 & -2.23 & 2.02 & $4.96 \times 10^{-6}$ & $4.04 \times 10^{-4}$ \\
\hline $\begin{array}{l}E 4-D 2-U 2 P \\
(60.8,0.53)\end{array}$ & 0.004 & 0.85 & -0.128 & -2.23 & 5.02 & $4.96 \times 10^{-6}$ & $2.51 \times 10^{-3}$ \\
\hline $\begin{array}{l}E 4-D 2-U 3 P \\
(58.1,0.865)\end{array}$ & 0.020 & 0.99 & -0.0133 & -2.23 & 2.17 & $4.97 \times 10^{-6}$ & $4.65 \times 10^{-4}$ \\
\hline $\begin{array}{l}E 4-D 2-U 4 P \\
(60.7,0.50)\end{array}$ & 0.003 & 0.85 & -0.109 & -2.23 & 5.30 & $4.97 \times 10^{-6}$ & $2.81 \times 10^{-3}$ \\
\hline $\begin{array}{l}E 4-D 4-U 1 P \\
(55.3,0.76)\end{array}$ & 0.014 & 0.96 & -1.09 & -2.49 & 2.09 & $5.01 \times 10^{-6}$ & $4.29 \times 10^{-4}$ \\
\hline $\begin{array}{l}E 4-D 4-U 2 P \\
(62.0,0.68)\end{array}$ & 0.019 & 0.89 & -1.49 & -2.37 & 4.16 & $3.39 \times 10^{-6}$ & $1.72 \times 10^{-3}$ \\
\hline $\begin{array}{l}E 4-D 4-U 3 P \\
(62.6,0.94)\end{array}$ & 0.020 & 0.97 & -1.08 & -2.49 & 2.24 & $5.01 \times 10^{-6}$ & $4.96 \times 10^{-4}$ \\
\hline $\begin{array}{l}E 4-D 4-U 4 P \\
(56.1,0.555)\end{array}$ & 0.018 & 0.89 & -1.45 & -2.34 & 4.26 & $3.36 \times 10^{-6}$ & $1.81 \times 10^{-3}$ \\
\hline
\end{tabular}

Then we have $\sin ^{2} 2 \theta_{e \mu}=0.0200$ and $\sin ^{2} 2 \theta_{\mu \tau}=0.990$. With $R=0.865 \times 10^{13}$, we found

$$
\begin{aligned}
& m_{1}=-1.33 \times 10^{-5} \mathrm{eV}, \quad m_{2}=-2.23 \times 10^{-3} \mathrm{eV}, \\
& m_{3}=2.17 \times 10^{-2} \mathrm{eV},
\end{aligned}
$$

which are multiplied by $0.865 \times 10^{13} / R$ if the $R$ dependence is kept. Thus

$$
\Delta m_{12}^{2}=4.97 \times 10^{-6} \mathrm{eV}^{2}, \quad \Delta m_{23}^{2}=4.05 \times 10^{-4} \mathrm{eV}^{2} .
$$

As we can see from Figs. 2(a), 2(b), and 2(c), the angle $\sin ^{2} 2 \theta_{\mu \tau}$ takes values larger than 0.7 for a wider range of $r$. The same holds for the ratio $\Delta m_{12}^{2} / \Delta m_{23}^{2}$. The allowed region of $r$ is essentially fixed by $\sin ^{2} 2 \theta_{e \mu}$. There are three allowed regions. This situation holds for all cases E2-D2-UiP, E2-D4-UiP, E4-D2-UiP, and E2-D4-UiP $(i=1-4)$. 


\section{2. $m_{b} m_{\tau}>0$ case}

a. E1-D3-U4M with $r=680$. The Dirac mass term and the Majorana mass term are given by

$$
\begin{aligned}
& \tilde{M}_{\nu D}=\left(\begin{array}{ccc}
-0.2089 & -4.124 & -1.638 \\
-4.124 & -29.95 & -22.15 \\
-1.638 & -22.15 & -11.74
\end{array}\right), \\
& \tilde{M}_{\nu R}=R\left(\begin{array}{lll}
0.0003 & 0.0061 & 0.0024 \\
0.0061 & 0.0445 & 0.0326 \\
0.0024 & 0.0326 & -0.172
\end{array}\right) .
\end{aligned}
$$

As we expected, both $M_{\nu D}$ and $M_{\nu R}$ have nonhierarchical forms for the part relevant to the second and third generations. The neutrino mass matrix and the neutrino mixing matrix are given by

$$
\begin{aligned}
& m_{\nu}=\frac{1}{R}\left(\begin{array}{ccc}
-141.3 & -2805 & -1111 \\
-2805 & -20159 & -15018 \\
-1111 & -15018 & -7336
\end{array}\right), \\
& U_{\nu}=\left(\begin{array}{ccc}
0.968 & 0.0485 & -0.246 \\
-0.231 & 0.553 & -0.800 \\
0.0973 & 0.831 & 0.547
\end{array}\right)
\end{aligned}
$$

Then we have $\sin ^{2} 2 \theta_{e \mu}=0.009$ and $\sin ^{2} 2 \theta_{\mu \tau}=0.92$. With $R=1.22 \times 10^{15}$, we found

$$
\begin{aligned}
& m_{1}=2.21 \times 10^{-7} \mathrm{eV}, \quad m_{2}=2.24 \times 10^{-3} \mathrm{eV}, \\
& m_{3}=-2.49 \times 10^{-2} \mathrm{eV} .
\end{aligned}
$$

Thus

$$
\Delta m_{12}^{2}=5.00 \times 10^{-6} \mathrm{eV}^{2}, \quad \Delta m_{23}^{2}=6.15 \times 10^{-4} \mathrm{eV}^{2} .
$$

As we can see from Figs. 3(a), 3(b), and 3(c), the angle $\sin ^{2} 2 \theta_{\mu \tau}$ takes values larger than 0.7 for a wider range of $r$. The same holds for the ratio $\Delta m_{12}^{2} / \Delta m_{23}^{2}$. The allowed region of $r$ is essentially fixed by $\sin ^{2} 2 \theta_{e \mu}$. There are three allowed regions similarly to the case given in Sec. III B 1 a. This situation holds for all cases E1-D3-UiM $(i=1-4)$.

\begin{tabular}{|c|c|c|c|c|c|}
\hline & & & (a) & & \\
\hline$E 1-D 1$ & $i M(i=$ & $, 2,3,4)$ & & & \\
\hline$e, \mu$ & $d, s$ & $u, c, t$ & $r$ & $R\left(10^{15}\right)(r)$ & $\sin ^{2} 2 \theta_{\mu \tau}$ \\
\hline$(-,-)$ & $(-,-)$ & $(-,-),-$ & $510-520,550,830-840,900-950$ & $0.55-0.99(550)$ & 0.92 \\
\hline & & $(-,+),-$ & $510-520,540,830-840,900-950$ & $1.30-2.36(830)$ & 0.92 \\
\hline & & $(+,-),-$ & $510-520,550,830-840,900-950$ & $0.55-0.99(550)$ & 0.92 \\
\hline & & $(+,+),-$ & $510-520,540,830-840,900-950$ & $1.30-2.36(830)$ & 0.92 \\
\hline$E 1-D 3$ & $i M(i=$ & $, 2,3,4)$ & & & \\
\hline$e, \mu$ & $d, s$ & $u, c, t$ & $r$ & $R\left(10^{15}\right)(r)$ & $\sin ^{2} 2 \theta_{\mu \tau}$ \\
\hline$(-,-)$ & $(+,-)$ & $(-,-),-$ & $550-580,640-740,840-910$ & $0.84-1.51(690)$ & 0.91 \\
\hline & & $(-,+),-$ & $560-580,640-740,840-900$ & $0.87-1.57(680)$ & 0.92 \\
\hline & & $(+,-),-$ & $550-580,640-740,840-910$ & $0.84-1.51(690)$ & 0.91 \\
\hline & & $(+,+),-$ & $560-580,640-740,840-900$ & $0.87-1.57(680)$ & 0.92 \\
\hline$E 3-D 1$ & $i M(i=$ & ,2,3,4) & & & \\
\hline$e, \mu$ & $d, s$ & $u, c, t$ & $r$ & $R\left(10^{15}\right)(r)$ & $\sin ^{2} 2 \theta_{\mu \tau}$ \\
\hline$(+,-)$ & $(-,-)$ & $(-,-),-$ & $510,540,840-850,910-960$ & $1.36-2.47(840)$ & 0.91 \\
\hline & & $(-,+),-$ & $510,540,840-850,910-950$ & $1.38-2.51(840)$ & 0.92 \\
\hline & & $(+,-),-$ & $510,540,840-850,910-960$ & $1.36-2.47(840)$ & 0.91 \\
\hline & & $(+,+),-$ & $510,540,840-850,910-950$ & $1.38-2.51(840)$ & 0.92 \\
\hline$E 3-D 3$ & $i M(i=$ & $, 2,3,4)$ & & & \\
\hline$e, \mu$ & $d, s$ & $u, c, t$ & $r$ & $R\left(10^{15}\right)(r)$ & $\sin ^{2} 2 \theta_{\mu \tau}$ \\
\hline$(+,-)$ & $(+,-)$ & $(-,-),-$ & $520-540,570-580,790-810,880-940$ & $1.12-2.04(790)$ & 0.92 \\
\hline & & $(-,+),-$ & $530,570-580,790-810,880-930$ & $0.63-1.13(580)$ & 0.93 \\
\hline & & $(+,-),-$ & $520-540,570-580,790-810,880-940$ & $1.12-2.04(790)$ & 0.92 \\
\hline & & $(+,+),-$ & $530,570-580,790-810,880-930$ & $0.63-1.13(580)$ & 0.93 \\
\hline$E 1-D 2$ & $2 P$ & & & & \\
\hline$e, \mu$ & $d, s$ & $u, c, t$ & $r$ & $R\left(10^{13}\right)(r)$ & $\sin ^{2} 2 \theta_{\mu \tau}$ \\
\hline$(-,-)$ & $(-,+)$ & $(-,+),+$ & $36.2-36.5$ & $7.74-7.96(36.5)$ & 0.75 \\
\hline
\end{tabular}

b. E3-D3-U3M with $r=790$. The Dirac mass term and the Majorana mass term are similar to the case given in Sec. III B 2 a, so that we only show the neutrino mass matrix and the neutrino mixing matrix

TABLE II. (a) Ranges of the parameters $r$ and $R$ to predict the desired neutrino mixings and masses for $m_{b} m_{\tau}>0$. (b) Predictions of neutrino mixing angles and masses for $m_{b} m_{\tau}>0$. All cases correspond to (a). 
TABLE II. (Continued).

(b)

\begin{tabular}{|c|c|c|c|c|c|c|c|}
\hline $\begin{array}{l}\text { Model } \\
\left(r, R / 10^{15}\right)\end{array}$ & $\sin ^{2} 2 \theta^{e \mu}$ & $\sin ^{2} 2 \theta_{\mu \tau}$ & $m_{1}\left(10^{-3} \mathrm{eV}\right)$ & $m_{2}\left(10^{-3} \mathrm{eV}\right)$ & $m_{3}\left(10^{-2} \mathrm{eV}\right)$ & $\Delta m_{12}^{2}\left(\mathrm{eV}^{2}\right)$ & $\Delta m_{23}^{2}\left(\mathrm{eV}^{2}\right)$ \\
\hline $\begin{array}{l}E 1-D 1-U 1 M \\
(550,0.77)\end{array}$ & 0.018 & 0.92 & 0.976 & 2.44 & -2.63 & $4.99 \times 10^{-6}$ & $6.90 \times 10^{-4}$ \\
\hline $\begin{array}{l}E 1-D 1-U 2 M \\
(830,1.83)\end{array}$ & 0.016 & 0.92 & 0.941 & 2.43 & -2.45 & $5.01 \times 10^{-6}$ & $5.96 \times 10^{-4}$ \\
\hline $\begin{array}{l}E 1-D 1-U 3 M \\
(550,0.77)\end{array}$ & 0.019 & 0.92 & 0.979 & 2.44 & -2.64 & $4.99 \times 10^{-6}$ & $6.90 \times 10^{-4}$ \\
\hline $\begin{array}{l}E 1-D 1-U 4 M \\
(830,1.83)\end{array}$ & 0.016 & 0.92 & 0.942 & 2.43 & -2.45 & $5.01 \times 10^{-6}$ & $5.96 \times 10^{-4}$ \\
\hline $\begin{array}{l}E 1-D 3-U 1 M \\
(690,1.175)\end{array}$ & 0.009 & 0.9 & $2.30 \times 10^{-4}$ & 2.24 & -2.72 & $5.00 \times 10^{-6}$ & $7.33 \times 10^{-4}$ \\
\hline $\begin{array}{l}E 1-D 3-U 2 M \\
(680,1.22)\end{array}$ & 0.009 & 0.92 & $2.34 \times 10^{-4}$ & 2.23 & -2.49 & $4.99 \times 10^{-6}$ & $6.15 \times 10^{-4}$ \\
\hline $\begin{array}{l}E 1-D 3-U 3 M \\
(690,1.175)\end{array}$ & 0.010 & 0.91 & $2.49 \times 10^{-4}$ & 2.24 & -2.72 & $5.01 \times 10^{-6}$ & $7.33 \times 10^{-4}$ \\
\hline $\begin{array}{l}E 1-D 3-U 4 M \\
(680,1.22)\end{array}$ & 0.009 & 0.92 & $2.21 \times 10^{-4}$ & 2.24 & -2.49 & $5.00 \times 10^{-6}$ & $6.15 \times 10^{-4}$ \\
\hline $\begin{array}{l}E 3-D 1-U 1 M \\
(840,1.915)\end{array}$ & 0.015 & 0.91 & 1.16 & 2.52 & -2.44 & $5.00 \times 10^{-6}$ & $5.91 \times 10^{-4}$ \\
\hline $\begin{array}{l}E 3-D 1-U 2 M \\
(840,1.945)\end{array}$ & 0.017 & 0.92 & 1.14 & 2.51 & -2.36 & $5.01 \times 10^{-6}$ & $5.50 \times 10^{-4}$ \\
\hline $\begin{array}{l}E 3-D 1-U 3 M \\
(840,1.915)\end{array}$ & 0.015 & 0.91 & 1.16 & 2.52 & -2.44 & $5.00 \times 10^{-6}$ & $5.91 \times 10^{-4}$ \\
\hline $\begin{array}{l}E 3-D 1-U 4 M \\
(840,1.945)\end{array}$ & 0.017 & 0.92 & 1.14 & 2.51 & -2.36 & $5.00 \times 10^{-6}$ & $5.50 \times 10^{-4}$ \\
\hline $\begin{array}{l}E 3-D 3-U 1 M \\
(790,1.58)\end{array}$ & 0.016 & 0.92 & 0.241 & 2.25 & -2.65 & $5.00 \times 10^{-6}$ & $6.96 \times 10^{-4}$ \\
\hline $\begin{array}{l}E 3-D 3-U 2 M \\
(580,0.88)\end{array}$ & 0.016 & 0.93 & 0.232 & 2.25 & -2.50 & $4.99 \times 10^{-6}$ & $6.19 \times 10^{-4}$ \\
\hline $\begin{array}{l}E 3-D 3-U 3 M \\
(790,1.58)\end{array}$ & 0.016 & 0.92 & 0.242 & 2.25 & -2.65 & $5.00 \times 10^{-6}$ & $6.96 \times 10^{-4}$ \\
\hline $\begin{array}{l}E 3-D 3-U 4 M \\
(580,0.88)\end{array}$ & 0.017 & 0.93 & 0.233 & 2.25 & -2.50 & $5.00 \times 10^{-6}$ & $6.19 \times 10^{-4}$ \\
\hline $\begin{array}{l}\text { Model } \\
\left(r, R / 10^{15}\right)\end{array}$ & $\sin ^{2} 2 \theta^{e \mu}$ & $\sin ^{2} 2 \theta_{\mu \tau}$ & $m_{1}\left(10^{-3} \mathrm{eV}\right)$ & $m_{2}\left(10^{-3} \mathrm{eV}\right)$ & $m_{3}\left(10^{-2} \mathrm{eV}\right)$ & $\Delta m_{12}^{2}\left(\mathrm{eV}^{2}\right)$ & $\Delta m_{23}^{2}\left(\mathrm{eV}^{2}\right)$ \\
\hline $\begin{array}{l}E 1-D 2-U 2 P \\
(36.5,0.0785)\end{array}$ & 0.006 & 0.75 & $-9.47 \times 10^{-4}$ & 3.12 & -1.47 & $9.71 \times 10^{-6}$ & $2.06 \times 10^{-4}$ \\
\hline
\end{tabular}

$$
\begin{aligned}
& m_{\nu}=\frac{1}{R}\left(\begin{array}{ccc}
214.7 & -3785 & -1502 \\
-3785 & -28242 & -20425 \\
-1502 & -20425 & -9864
\end{array}\right), \\
& U_{\nu}=\left(\begin{array}{ccc}
0.962 & 0.0630 & -0.267 \\
-0.257 & 0.544 & -0.799 \\
0.0946 & 0.837 & 0.539
\end{array}\right) .
\end{aligned}
$$

As we can see from Figs. 4(a), 4(b), and 4(c), the angle $\sin ^{2} 2 \theta_{\mu \tau}$ takes values larger than 0.7 for a wider range of $r$. The same holds for the ratio $\Delta m_{12}^{2} / \Delta m_{23}^{2}$. The allowed region of $r$ is essentially fixed by $\sin ^{2} 2 \theta_{e \mu}$. There are two allowed regions. This situation holds for all cases E1-D1-UiM, E3-D1-UiM, and E3-D3-UiM ( $i=1-4)$.

c. $E 1-D 2-U 2 P$ with $r=36.5$. This is a very special case corresponding to the case given in Sec. II E 2 b. The Dirac mass term and the Majorana mass term are given by

$\widetilde{M}_{\nu D}=\left(\begin{array}{ccc}-0.01399 & 0.21534 & -0.10180 \\ 0.21534 & -3.1557 & -1.1315 \\ -0.10180 & -1.1315 & 135.29\end{array}\right)$, 


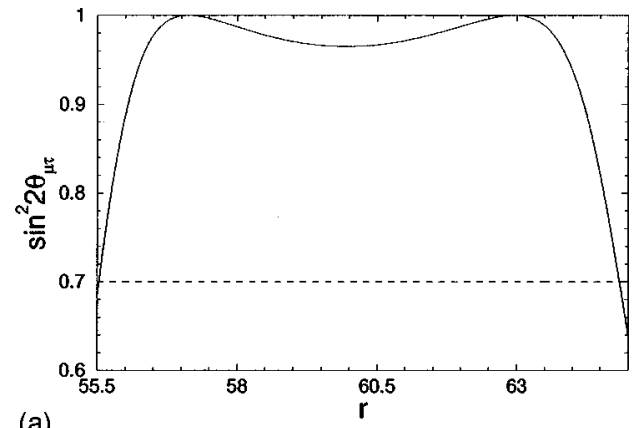

(a)
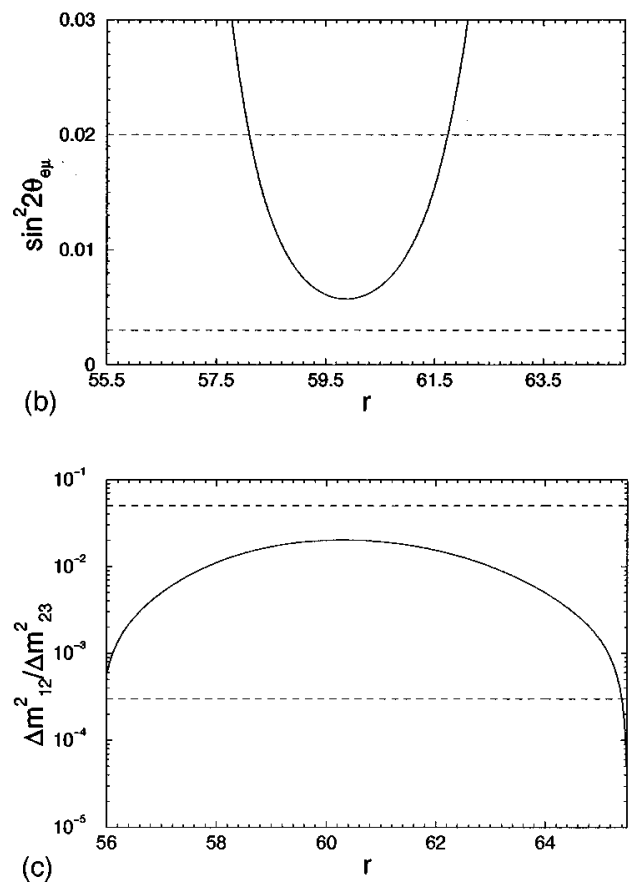

FIG. 1. Parameter $r$ dependence of neutrino mixing angles and the ratio of neutrino mass squared differences for the $E 1-D 3-U 2 P$ case $\left(m_{b} m_{r}<0\right)$. (a), (b), and (c) show the $r$ dependence of $\sin ^{2} 2 \theta_{\mu \tau}, \sin ^{2} 2 \theta_{e \mu}$, and $\Delta m_{12}^{2} / \Delta m_{23}^{2}$. The allowed region of $r$ is essentially determined by reproducing $\sin ^{2} 2 \theta_{e \mu}$.

As we expected, $M_{\nu D}$ has a hierarchical form for the part relevant to the second and third generations, while $M_{\nu R}$ is less hierarchical, because of the cancellation of the 33 element in $M_{\nu R}$. The neutrino mass matrix and the neutrino mixing matrix are given by

$$
\begin{gathered}
m_{\nu}=\frac{1}{R}\left(\begin{array}{ccc}
-0.5586 & 8.014 & 27.97 \\
8.014 & -107.5 & -606.3 \\
27.97 & -606.3 & -800.1
\end{array}\right), \\
U_{\nu}=\left(\begin{array}{ccc}
0.999 & 0.0374 & -0.00658 \\
-0.0290 & 0.864 & -0.502 \\
-0.007 & 0.502 & 0.865
\end{array}\right) .
\end{gathered}
$$

Then we have $\sin ^{2} 2 \theta_{e \mu}=0.006$ and $\sin ^{2} 2 \theta_{\mu \tau}=0.75$. With $R=7.85 \times 10^{13}$, we found

$$
m_{1}=-9.47 \times 10^{-7} \mathrm{eV}, \quad m_{2}=3.12 \times 10^{-3} \mathrm{eV},
$$
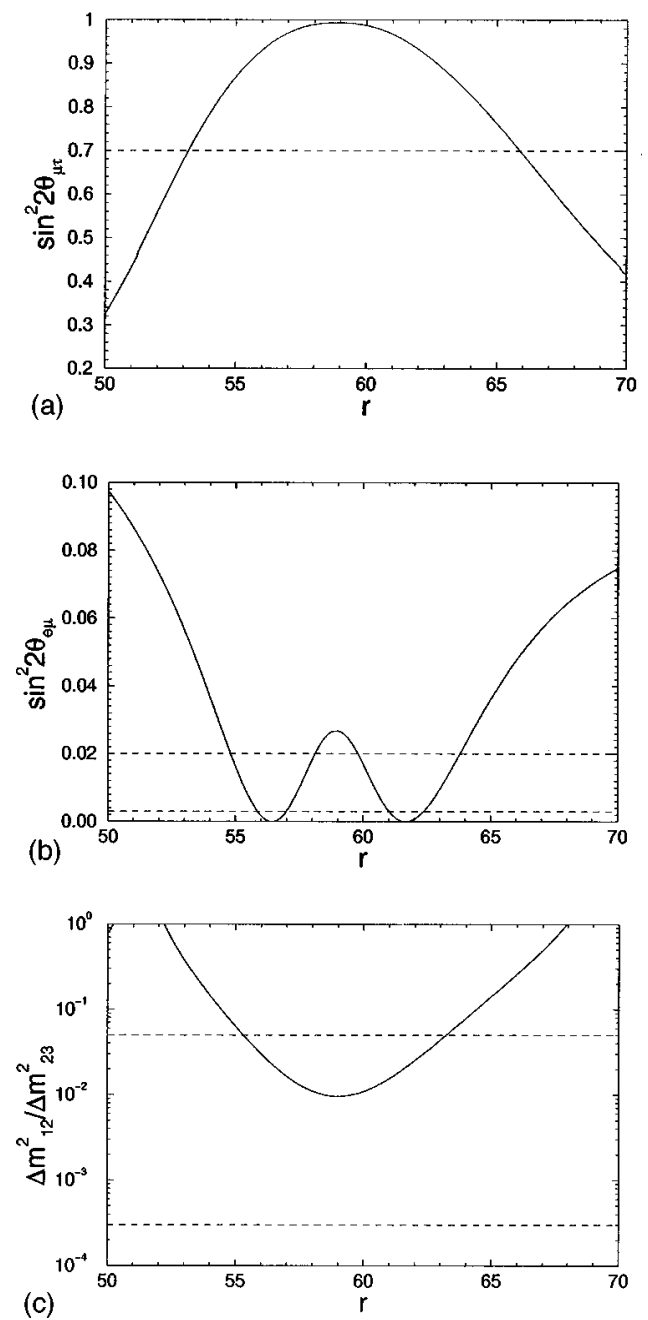

FIG. 2. Same as Fig. 1 for the E4-D2-U3P case $\left(m_{b} m_{r}<0\right)$. The allowed region of $r$ is essentially determined by reproducing $\sin ^{2} 2 \theta_{e \mu}$.

$$
m_{3}=-1.47 \times 10^{-2} \mathrm{eV} .
$$

Thus

$$
\Delta m_{12}^{2}=9.71 \times 10^{-6} \mathrm{eV}^{2}, \quad \Delta m_{23}^{2}=2.06 \times 10^{-4} \mathrm{eV}^{2} .
$$

As we can see from Figs. 5(a), 5(b), and 5(c), $\sin ^{2} 2 \theta_{e \mu}$ is insensitive to $r$ and the allowed region is determined by $\sin ^{2} 2 \theta_{\mu \tau}$ and $\Delta m_{12}^{2} / \Delta m_{23}^{2}$. The allowed region of $r$ is a tiny region, so that the solution is quite sensitive to the value of $r$. This case will be the most unlikely one.

Finally, we comment on the CHOOZ data [2]. Since $\Delta m_{12}^{2} \ll \Delta m_{23}^{2}$, we can consider the mixing angle for the CHOOZ disappearance test of $\nu_{e}$ may be defined by $\sin \theta_{e X}$ $=\left(U_{\nu}\right)_{13}$, which is $\sin \theta_{e \tau}$ in our cases and the mass-squared difference is $\Delta m^{2} \simeq m_{\nu \tau}^{2} \equiv m_{3}^{2}$. Then we compared these values of parameters with the $\mathrm{CHOOZ}$ two-neutrino analysis. We found the following: All cases except $E 1-D 2-U 2 P$ predict $\sin ^{2} 2 \theta_{e \tau} \sim(0.2-0.3)$. For $m_{b} m_{\tau}<0$ with $m_{\mu} m_{c}<0$ cases and also $m_{b} m_{\tau}>0$ cases, we predict $\Delta m^{2}$ $<10^{-3} \mathrm{eV}^{2}$, so that they are safe. For $m_{b} m_{\tau}<0$ with 

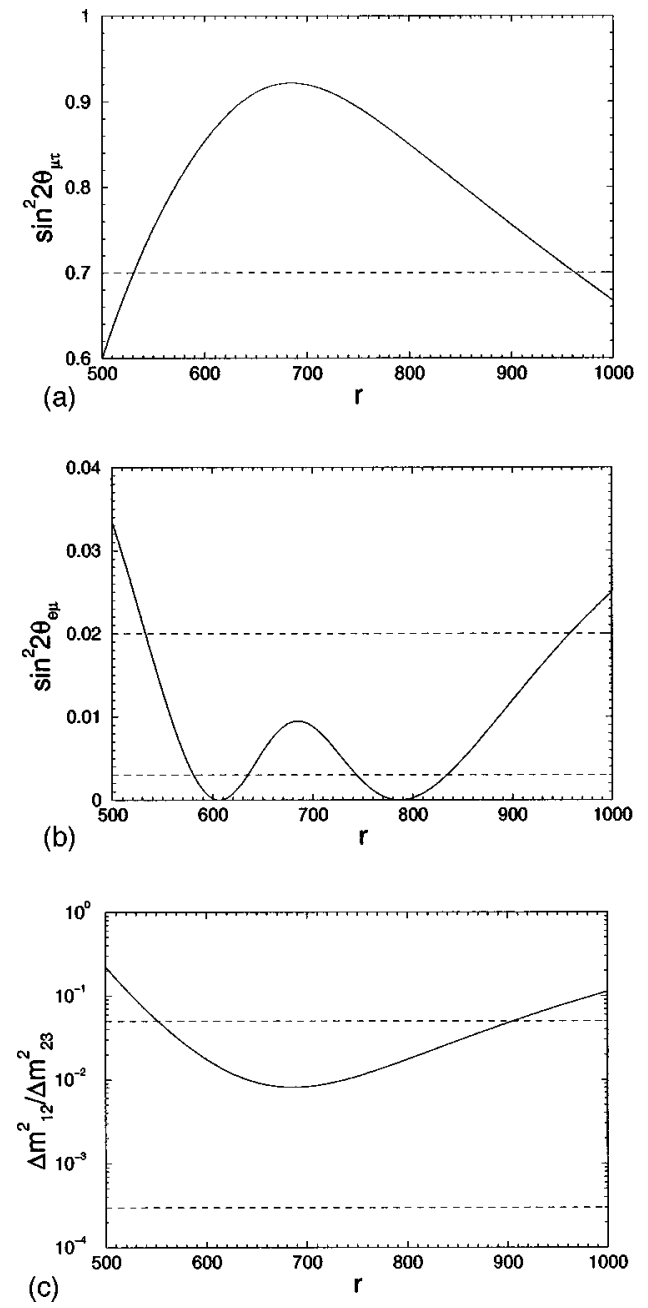

FIG. 3. Same as Fig. 1 for the E1-D3-U4M case $\left(m_{b} m_{\tau}\right.$ $>0$ ). The allowed region of $r$ is essentially determined by reproducing $\sin ^{2} 2 \theta_{e \mu}$.

$m_{\mu} m_{c}>0$ cases, we predict $\Delta m^{2} \sim 2 \times 10^{-3} \mathrm{eV}^{2}$ and their values are on the boundary of the excluded region. This can be remedied by changing $r$ slightly from the value we took to achieve the largest value of $\sin ^{2} 2 \theta_{\mu \tau}$ or by taking a larger value of $R$ to reduce the overall neutrino mass scale. For the $E 1-D 2-U 2 P$ case, we predict $\sin ^{2} 2 \theta_{e \tau} \sim 1 \times 10^{-4}$, so that this satisfies the bound. In summary, many of our cases predict in general rather large values of $\left(U_{\nu}\right)_{13} \sim 0.25$. However, all cases satisfy the $\mathrm{CHOOZ}$ bound. In several cases corresponding to $m_{b} m_{\tau}<0$ with $m_{\mu} m_{c}>0$, the $\nu_{e}-\nu_{\tau}$ oscillation is large enough to be observed in the near future experiments.

We explicitly showed some of our solutions. Other cases show the similar matrices to one of the above cases. For their cases, we only showed neutrino mass and mixing in Tables I and II.

\section{SUMMARY}

We showed the mechanism to induce the nonhierarchical neutrino mass matrix by using the hierarchical forms of mass
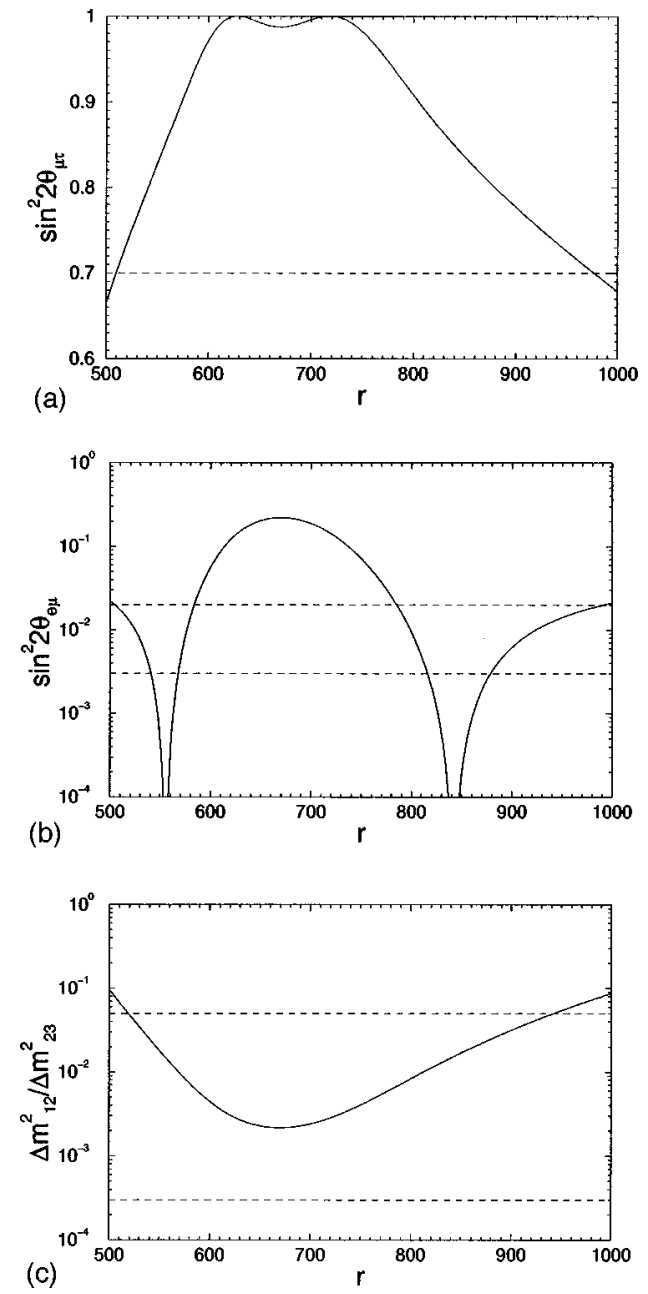

FIG. 4. Same as Fig. 1 for the E3-D3-U3M case $\left(m_{b} m_{\tau}>0\right)$. The allowed region of $r$ is essentially determined by reproducing $\sin ^{2} 2 \theta_{e \mu}$

matrices of $\widetilde{M}_{u}, \widetilde{M}_{d}$, and $\widetilde{M}_{l}$, although it is a quite nontrivial problem. Our model contains only one adjustable parameter $r$ which determines neutrino mixings and the ratios of neutrino masses. The other parameter $R$ is used to determine the absolute magnitude of neutrino masses, so that it determines $m_{\nu \tau}$ in our hierarchical mass spectrum of neutrinos.

Following our mechanism, we found many solutions, which are classified into three cases. Our mechanism guarantees the nonhierarchical structure only for the part relevant to the second and third generations and thus we inevitably predict small mixing between $\nu_{e}$ and $\nu_{\mu}$, while large mixing between $\nu_{\mu}$ and $\nu_{\tau}$. We examined the $r$ dependence of mixing angles and the ratios of neutrino masses and showed the sensitivity of these quantities to $r$. Depending on the choice of relative signs of fermion masses, the pattern of sensitivity changed. We showed that the solutions are not very sensitive to $r$ except the $E 1-D 2-U 2 P$ case.

There arises the question of whether the existence of the solutions depends crucially on the values of quark masses and mixings and/or the value of $\tan \beta$. We examined another set of the values used by Chacko and Mohapatra [8] and 

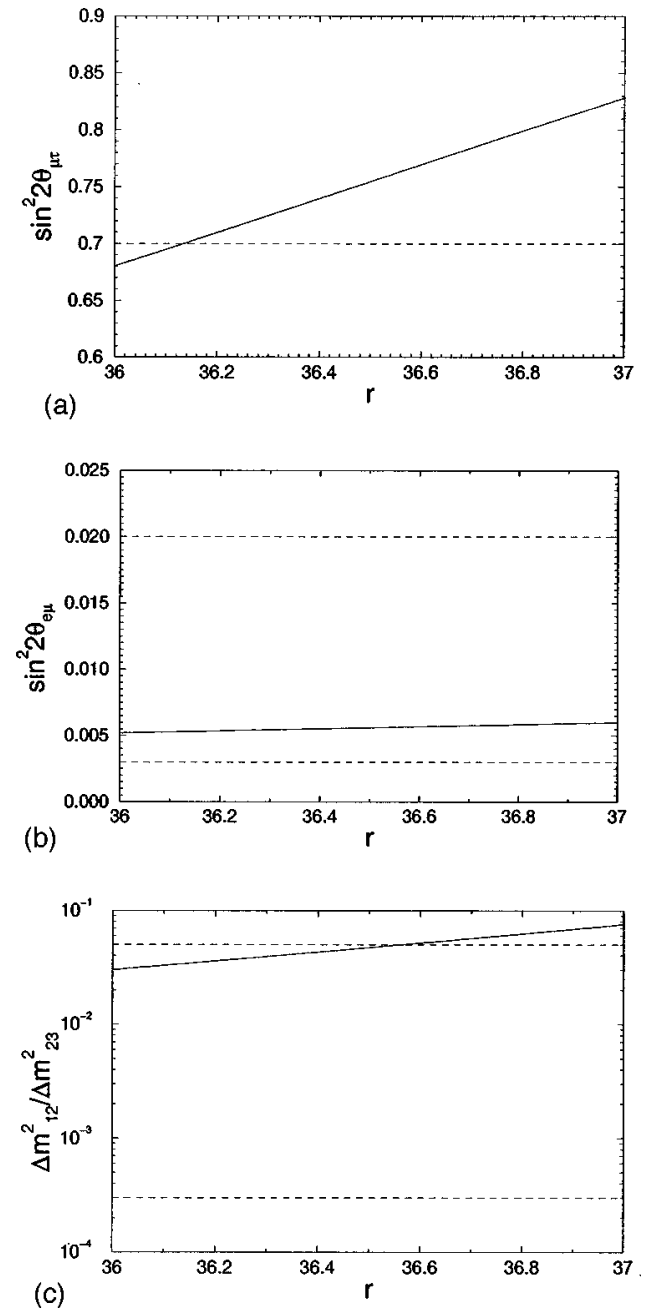

FIG. 5. Same as Fig. 1 for the $E 1-D 2-U 2 P$ case $\left(m_{b} m_{\tau}>0\right)$. The allowed region of $r$ is essentially determined by reproducing $\sin ^{2} 2 \theta_{\mu \tau}$ and $\Delta m_{12}^{2} / \Delta m_{23}^{2}$. The allowed region is a tiny one, so that we need a fine-tuning of $r$.

found that the solutions given in this paper exist with the small change of the values of $r$ and $R$. Thus the existence of our solutions does not depend on them.

Finally, we comment on $\mathrm{CP}$ violation. In the present analysis, we ignored $\mathrm{CP}$ violation effects. When $\mathrm{CP}$ violation turns on, many phases enter in our model. One is from the Kobayashi-Maskawa phase and others are from the phases of quark and charged lepton masses. These phases will relax the tight situation which we considered. Thus we expect that with $\mathrm{CP}$ violation models cover the broader range of the mixing angles and the neutrino masses. This problem is under consideration.

Also, there is no good reason to assume that $\widetilde{M}_{l}$ is diagonal, although it would be a hierarchical form. If we relax this assumption, the models would cover a wider range of mixings and masses than what we obtained in this paper.

\section{ACKNOWLEDGMENTS}

This work is supported in part by the Japanese Grant-inAid for Scientific Research of Ministry of Education, Science, Sports and Culture, No. 08640374 and No. 10140216.

\section{APPENDIX: THE PROOF OF THE INCOMPATIBILITY OF THE MINIMAL MODEL AND QUARK AND CHARGED LEPTON MASS SPECTRUM}

For one ten-dimensional Higgs field $n=1$ case, we have an additional relation

$$
\widetilde{M}_{l}=c_{u} \widetilde{D}_{u}+c_{d} \widetilde{M}_{d},
$$

where

$$
c_{u}=\left(1-\frac{4}{1-\frac{\kappa_{d}}{\kappa_{u}} \frac{v_{u}}{v_{d}}}\right), \quad c_{d}=4\left(\frac{\frac{v_{u}}{v_{d}}}{1-\frac{\kappa_{d}}{\kappa_{u}} \frac{v_{u}}{v_{d}}}\right) .
$$

In this appendix, we first discuss that the model containing one 10 and one 126 representation cannot reproduce the charged lepton masses and thus the model is rejected independently of neutrino masses and mixings. Then we go to the model which contains $n(\geqslant 2) 10$ and one 126 models. We consider the ordinary seesaw mechanism to derive the lefthanded neutrino mass matrix. In the case of the type-II seesaw model proposed by Mohapatra and Senjanovic [11], there appears the left-handed neutrino mass matrix. In our case, we do not consider this by taking $v_{L}$ is small.

This model was considered by Mohapatra and co-workers $[4,5,8]$ as a model to give a unified model to explain quark and lepton masses and mixings. Recently, Brahmachari and Mohapatra [8] showed that this model cannot explain the desired neutrino mixing pattern, the small mixing between $\nu_{e}$ and $\nu_{\mu}$, and the large mixing between $\nu_{\mu}$ and $\nu_{\tau}$.

Here we show that this model is not able to explain the charged lepton masses, so that the model is rejected independently of the arguments on neutrino masses and mixings. First, we see that the charged lepton mass matrix is written in a linear combination of quark mass matrices as in Eq. (A1).

Because $\left|m_{\tau}\right| \simeq\left|m_{b}\right| \sim \lambda^{3}\left|m_{t}\right|$ with the Cabibbo angle $\lambda$ $=0.2205, c_{u} \sim \lambda^{3}$ and $c_{d} \sim 1$. Knowing that $\widetilde{D}_{u}$ is a diagonal matrix and $\widetilde{M}_{d}$ is almost diagonal, and also the mass hierarchy of the $u$-quark sector is much severer that that of the $d$-quark sector, we observe that the contribution to the first and second generation parts of $\widetilde{M}_{l}$ from the $u$-quark part $D_{u}$ is negligible so that it is proportional to that of $\widetilde{M}_{d}$. Thus the mass matrix that predicts $m_{e} / m_{\mu} \simeq m_{d} / m_{s}$ does not reproduce the observed hierarchical structure of the $d$-quark and charged lepton masses such as predicted by the GeorgiJarskog mass relations $m_{b}=m_{\tau}, m_{s}=m_{\mu} / 3$, and $m_{d}=3 m_{e}$ at the GUT scale. 
[1] T. Kajita, talk given at Neutrino 98, Takayama, 1998; SuperKamiokunde collaboration, Y. Fukuda et al., Phys. Rev. Lett. 81, 1562 (1998).

[2] C. Bemporad, talk given at Neutrino 98, [1]; Phys. Lett. B 420, 397 (1998).

[3] Y. Suzuki, talk given at Neutrino 98 [1].

[4] K. S. Babu and R. N. Mohapatra, Phys. Rev. Lett. 70, 2845 (1993).

[5] D-G. Lee and R. N. Mohapatra, Phys. Rev. D 51, 1353 (1995).
[6] K. S. Babu and Q. Shafi, Phys. Lett. B 294, 235 (1992).

[7] Y. Achiman and T. Greiner, Phys. Lett. B 329, 33 (1994); Nucl. Phys. B443, 3 (1995).

[8] B. Brahmachari and R. N. Mohapatra, Phys. Rev. D 58, 015001 (1998).

[9] H. Fusaoka and Y. Koide, Phys. Rev. D 57, 3986 (1998).

[10] N. Hata and P. Langacker, Phys. Rev. D 56, 6107 (1997).

[11] R. N. Mohapatra and G. Senjanovic, Phys. Rev. D 23, 165 (1981). 